\title{
Videoprojekt „Mein geheimes Porto“: Anwendung eines Modells für handlungsorientiertes Lernen im universitären Kontext
}

\author{
Anette Kind \\ Faculdade de Letras da Universidade do Porto \\ Centro de Linguística da Universidade do Porto, Portugal \\ akind@letras.up.pt \\ Ingrid Scholz \\ Philologisch-Historische Fakultät, Universität Augsburg, Deutschland \\ Ingrid.scholz@philhist.uni-augsburg.de \\ Simone Auf der Maur Tomé \\ Faculdade de Letras da Universidade do Porto \\ Centro de Linguística da Universidade do Porto, Portugal \\ stome@letras.up.pt
}

\begin{abstract}
2017 wurde Porto zum dritten Mal in der Online-Abstimmung European Best Destinations zum beliebtesten Reiseziel Europas gewählt. Vor diesem Hintergrund wurden im Studienjahr 2016/2017 alle Studierenden der germanistischen Studiengänge der Universität Porto zur Teilnahme an einem Videoprojekt eingeladen. Ziel des Projekts bestand darin, versteckte Winkel ihrer Universitätsstadt zu entdecken und diese aus persönlicher Sicht jenseits des touristischen Mainstreams in selbstproduzierten Kurzvideos in deutscher Sprache zu präsentieren.

Das Projekt sollte den Studierenden die Möglichkeit bieten, einerseits fremdsprachliche und mediale Kompetenzen gesamtheitlich und ziel- und handlungsorientiert anzuwenden, sich andererseits darin eigeninitiativ und konstruktiv einzubringen. Durch die subjektive inhaltliche Relevanz, die Möglichkeit der medialen Partizipation - durch Online-Veröffentlichung der Projektergebnisse - und nicht zuletzt durch die Aussicht auf eine Prämierung wurde eine hohe Resonanz angestrebt, was sich dann auch in der Teilnehmer*innenzahl widerspiegelte.

Vor dem Hintergrund einer kommunikativen Fremdsprachendidaktik möchten wir mit diesem Artikel aufzeigen, inwiefern durch eine handlungs- und aufgabenorientierte Vorgehensweise im universitären Fremdsprachenunterricht ein Beitrag zur Förderung
\end{abstract}


KIND, Anette; Scholz, Ingrid; Auf Der Maur Tomé, Simone, - Videoprojekt:..

Para lá da tarefa: implicar os estudantes na aprendizagem de línguas estrangeiras no ensino superior. Porto: FLUP, 2019, pp. 226-244 DOI: https://doi.org/10.21747/9789898969217/paraa12

selbstverantwortlicher Lernprozesse und fremdsprachlichen Handelns durch einen konstruktiven, kreativen und produktiven Umgang mit digitalen Ressourcen in einem authentischen Szenario geleistet werden kann.

Schlüsse/wörter: Projektarbeit, Deutsch als Fremdsprache, handlungsorientiertes Lernen, universitärer Fremdsprachenunterricht, digitales Lernen, Videoprojekt

\begin{abstract}
In 2017, Porto was voted Europe's most popular tourist destination for the third time in the European Best Destinations online poll. Against this background, in the academic year 2016/2017, all students of German Studies at the University of Porto were invited to participate in a video project. The aim of the project was to discover hidden corners of their university city and to present them from a personal point of view beyond the tourist mainstream through self-produced short videos in German.

The project was intended to offer students the opportunity to apply foreign-language and media-related competences in a holistic, goal-oriented and action-oriented manner. In addition, students were invited to take the initiative, making an individual and constructive contribution. Due to the subjective content relevance, the possibility of media participation - by publishing the project results online - and not least by the prospect of an award, a high level of response was sought. This was reflected in the considerable number of participants.

Within a framework of communicative approaches to foreign language teaching, we would like to use this article to show to what extent a contribution to the promotion of self-reliant learning processes and foreign-language tasks through a constructive, creative and productive use of digital resources in an authentic scenario is achieved, through an action-oriented and task-oriented approach in university language teaching.
\end{abstract}

Keywords: Project work, German as a foreign language, task-based learning, university foreign language teaching, digital learning, video project

\title{
1 - Einleitung: Konzept und Zielsetzung
}

Dass die universitären Fremdsprachenkurse, in denen in einem engen Zeitplan durch die Curricula festgelegte, am Europäischen Referenzrahmen orientierte Niveaustufen mit den entsprechenden Kompetenzen zu erreichen sind, wenig Raum für eine kreative 
KIND, Anette; Scholz, Ingrid; Auf Der Maur Tomé, Simone, - Videoprojekt:..

Para lá da tarefa: implicar os estudantes na aprendizagem de línguas estrangeiras no ensino superior.

Porto: FLUP, 2019, pp. 226-244

DOI: https://doi.org/10.21747/9789898969217/paraa12

und praxisorientierte Anwendung der Sprachkenntnisse lassen, ist sicherlich kein spezifisches Phänomen der Deutschkurse an der Universität Porto. Vor diesem Hintergrund entstand der Gedanke, ein niveauübergreifendes und aus dem Sprachunterricht ausgelagertes Projekt anzubieten, das den Studierenden die Möglichkeit bot, die verschiedenen Fertigkeiten kombiniert anzuwenden, im Team zu arbeiten, sich individuell und kreativ einzubringen und am Ende ein originelles und vorzeigbares Ergebnis präsentieren zu können, das über den unterrichts- und fakultätsinternen Kontext hinaus auf diversen Plattformen veröffentlicht werden sollte. Wir versprachen uns von diesem Projekt, dass die Studierenden mit mehr Motivation und Eigeninitiative ihre Deutschkenntnisse anwenden und durch die Arbeit am Projekt auch erweitern würden.

Als für unsere Zielsetzung geeignete Form wählten wir das Kurzvideo, da dieses eine ganzheitliche Herangehensweise des Konzepts für den Filmbeitrag als auch der eingesetzten sprachlichen Kompetenzen erlaubt und des Weiteren die Kompetenzen der Studierenden im Umgang mit digitalen Medien fördert. ${ }^{1}$

Ein kurzer Überblick soll veranschaulichen, unter welchen Voraussetzungen das hier dargestellte Projekt durchgeführt wurde. An der Universität Porto ist Deutsch als Fremdsprache fester curricularer Bestandteil in den Bachelorstudiengängen Internationale Beziehungen, Angewandte Sprachen und Sprachen, Literatur- und Kulturwissenschaft sowie in den Masterstudiengängen Fremdsprachendidaktik und Übersetzung und Sprachdienstleistungen. Die DaF-Kurse werden in allen Semestern der o.g. Studiengänge in zwei Doppelstunden pro Woche auf den Niveaustufen A1 bis C1 durchgeführt. Die Mehrheit der Studierenden beginnt im 1. Semester des Bachelors mit A1, da der Deutschunterricht an den Sekundarschulen in den letzten Jahren kontinuierlich zurückgegangen ist und daher nur wenige Studienanfänger Vorkenntnisse mitbringen.

Alle Studierenden der germanistischen Studiengänge wurden dazu angeregt, versteckte Winkel der Stadt zu entdecken und dabei - auf Deutsch - originelle Kurzvideos zu produzieren, um Porto aus einer ganz persönlichen Sicht in seiner Vielfalt weit ab vom touristischen Blick darzustellen.

In diesem Projekt interessierte uns also das Porto jenseits des Mainstreams, jenseits der Besucherströme, die sich durch die Altstadt drängeln, d.h. der individuelle,

\footnotetext{
1 Das Projekt - in Form eines Videowettbewerbs - wurde im 2. Semester des Studienjahres 2016/2017 von den Lektorinnen der Germanistischen Abteilung, Anette Kind, Ingrid Scholz und Simone Tomé, konzipiert und koordiniert und über eine Projektpauschale des DAAD (Deutscher Akademischer Austauschdienst) finanziert.
} 
KIND, Anette; Scholz, Ingrid; Auf Der Maur Tomé, Simone, - Videoprojekt:..

Para lá da tarefa: implicar os estudantes na aprendizagem de línguas estrangeiras no ensino superior. Porto: FLUP, 2019, pp. 226-244 DOI: https://doi.org/10.21747/9789898969217/paraa12

unverstellte Blick auf die Kuriositäten der Stadt, etwa auf ein bizarres architektonisches Detail, auf einen verwunschenen, verwilderten Garten oder alternativen Laden, auf eine schräge Galerie oder verborgene Gasse, auf eine verfallene Fabrik von Anno dazumal, eine skurrile Kneipe oder eine althergebrachte Mercearia (Tante-Emma-Laden) oder auf ein anderes originelles Fundstück, das in gängigen Reiseführern oder auf Internetseiten keine Erwähnung findet.

Ferner zielte das Projekt darauf ab, einen Beitrag zur Entwicklung eines positiveren Bildes der deutschen Sprache zu leisten, die seitens unserer Studierenden oft vor und während des Lernprozesses mit gängigen Klischees verbunden, daher als schwierig, grammatiklastig und unschön klingend beschrieben wird. Das Projekt sollte also dazu dienen, dieser tendenziell negativen affektiven Einstellung entgegenzuwirken. Betrachtet man Emotionen als „Schlüssel zum Erfolg“ (Apeltauer 1997, p. 11) und integralen Bestandteil des Lernprozesses, der „aus der Außenperspektive besonders stark mit Gefühlen besetzt ist“" (Schwerdtfeger, 1997, p. 587), so liegt die Annahme nahe, dass sie einen direkten oder indirekten Einfluss auf die Auswahl der zu erlernenden Fremdsprache bzw. auf den gesamten Lernprozess und -erfolg derselben haben. Für Kleppin (2004, p. 3) ist die Motivation „eine vorhandene positive Einstellung zur Zielsprache bzw. vorhandene Gründe und Ursachen für das Erlernen einer Fremdsprache“.

In allen Projektphasen stand eine handlungsfundierte Auseinandersetzung mit der zu erlernenden Sprache Deutsch im Fokus. Die Fremdsprache sollte im Sinne von Ehlich (2007, p. 14) als Mittel fungieren, um Handlungen zu vollziehen. Vorhandene Sprachkenntnisse sollten dabei funktional-pragmatisch, d.h. an einen klaren Zweck gebunden eingesetzt und erweitert werden. In ihrer Studie zum fremdsprachlichen Handeln bzw. zur sprachlichen Tätigkeit in Projekten stellt Peuschel (2012, p. 49) dar, wie diese das Erlernen der Fremdsprache fördern können. Dabei unterscheidet sie zwischen sprachlichem Handeln mit kommunikativem Charakter und sprachlichem Handeln mit sprachbezogenem Charakter. Neben dem Sprachgebrauch und der Kommunikationstätigkeit sind laut Peuschel auch die notwendige Überarbeitung und die Anpassung bzw. die Modellierung der sprachlichen Äußerungen mit dem Ziel authentischen Kommunizierens für die Erstellung der Projektergebnisse entscheidend (ebd.).

Neben der Förderung kommunikativer Kompetenzen und sprachlichen Handelns beabsichtigten wir mit diesem Videoprojekt auch die Förderung medialer Kompetenz und Aktivität vor dem Hintergrund eines produktiven und ergebnisorientierten Umgangs mit 
KIND, Anette; Scholz, Ingrid; Auf Der Maur Tomé, Simone, - Videoprojekt:.. Para lá da tarefa: implicar os estudantes na aprendizagem de línguas estrangeiras no ensino superior. Porto: FLUP, 2019, pp. 226-244 DOI: https://doi.org/10.21747/9789898969217/paraa12

digitalen Medien in einem für den Lernenden begründeten Kontext. Hinsichtlich der Medienkompetenz unserer Studierenden sei angemerkt, dass sie in ihrem ersten Studiensemester das Fach Elektronische Ressourcen und Methoden des wissenschaftlichen Arbeitens belegen, in dem sie u.a. den Umgang mit digitalen Recherchetechniken, Datenbanken, Suchmaschinen, Repositorien, bibliographischen Normen, digitalen Wörterbüchern und Anwendungen für automatische Übersetzung sowie mit der Plattform für digitale Bildung Moodle erlernen. Darüber hinaus sind sie mit Office (insb. Word und Powerpoint) vertraut. Die Erstellung und die Bearbeitung von selbstgedrehten Videos finden hingegen keine explizite Berücksichtigung in den Studienplänen.

Baacke (1996, p. 8), der als Vater der Medienkompetenz gilt, definiert Medienkompetenz „als die Fähigkeit, in die Welt aktiv aneignender Weise auch alle Arten von Medien für das Kommunikations- und Handlungsrepertoire von Menschen einzusetzen“. Für Baacke ist

jeder Mensch prinzipiell ,mündiger Rezipient', er ist aber zugleich als kommunikativ-kompetentes Lebewesen auch ein aktiver Mediennutzer, muss also in der Lage sein [...], sich über die Medien auszudrücken. Dies muss geübt und gelernt werden, aber wir können solche Prozesse mit der Zuversicht beginnen, dass sie auch zu einem sinnvollen Ziele führen. (1996, p. 7)

Die Stärkung von Medienkompetenz bzw. die Förderung aktiver Medienarbeit lässt sich Baacke zufolge ausschließlich über Projektarbeit umsetzen (Baacke 1999, p. 35). Im hier beschriebenen Videoprojekt wurden die Studierenden durch die Veranstaltung eines Workshops darin unterstützt, vorhandene Fertigkeiten im Umgang mit digitalen Medien zu erweitern und in einem für sie relevanten und authentischen Szenario einzusetzen.

\section{2 - Projektarbeit und handlungsorientiertes Fremdsprachenlernen im universitären Kontext}

Projektarbeit im fremdsprachlichen Unterricht ist nichts Neues, sondern eine Weiterentwicklung des kommunikativ-interkulturellen Ansatzes (Funk et al., 2014, p. 129) und kann auf vielfältige Art und Weise umgesetzt werden. Ebenso vielfältig sind die in der Literatur vorliegenden Termini für die Bezeichnung von Projektunterricht und/oder -arbeit. Dennoch besteht Konsens darüber, dass durch Projektarbeit „das Lernen durch 
KIND, Anette; Scholz, Ingrid; Auf Der Maur Tomé, Simone, - Videoprojekt:.. Para lá da tarefa: implicar os estudantes na aprendizagem de línguas estrangeiras no ensino superior. Porto: FLUP, 2019, pp. 226-244 DOI: https://doi.org/10.21747/9789898969217/paraa12

eine handelnde, verschiedene Fertigkeiten integrierende Auseinandersetzung mit komplexen Situationen begünstigt wird" (Krumm et al., 2010, p. 1172). In der Fremdsprachendidaktik versteht man Projektunterricht als eine „offene und themenzentrierte Unterrichtsform, die ein hohes $\mathrm{Maß}$ an Selbstverantwortung und Mitbestimmung der Lernenden erlaubt“" (Legutke, 2003, p. 259).

Parallel zur Diskussion zur Projektarbeit im Unterricht entwickelte sich ab den 1980er Jahren maßgeblich durch die die Begrifflichkeiten prägenden Arbeiten von Gudjon \& Meyer der Ansatz des handlungsorientierten Unterrichts als ganzheitlicher und integrativer Lernform. Darin ist der Bezug des zu Erlernenden bzw. des Gelernten in der Umwelt der Lernenden, die zur Handlung herausgefordert werden, um Lösungen zu finden, verankert (vgl. Gudjon, 2001). Im Kontext des universitären Fremdsprachenunterrichts kann darauf basierend ein handlungsorientierter Lernansatz dazu dienen, den Lernenden Fertigkeiten zu vermitteln, die sie für ihre beruflich, wissenschaftlich oder persönlich relevanten Kontexte benötigen. Handlungsfordernde Aktivitäten werden aus lernpsychologischer Sicht als Auslöser von Aneignungsprozessen verstanden: In Handlungen besteht die Grundlage kognitiver Prozesse, indem das Lernen an einen konstruktiven, aktiven Prozess vonseiten der Lernenden gekoppelt wird. In Bezug auf den Zusammenhang von handlungsorientiertem Lernen in einem multimedialen Kontext betonen Heinz \& Gräsel schon Ende der 1990er Jahre, dass "Lernen immer eine aktive Beteiligung des Lernenden erfordert. Er muss sein Vorwissen auf die Aufgabe beziehen und die neue Information mit den bestehenden Kenntnissen in Übereinstimmung bringen" (Heinz \& Gräsel, 1997, p. 176). Dieses Postulat hat auch heute noch seine Gültigkeit.

Im Rahmen des in diesem Beitrag beschriebenen Videoprojekts ließ sich ein Lernszenario gestalten, das den von Gudjons und Meyer beschriebenen Prämissen handlungsorientierten Lernens Rechnung trägt, und zwar durch (vgl. Gudjon 2001, Jank \& Meyer, 2004):

a) eine ganzheitliche Auseinandersetzung der Lernenden mit der Wirklichkeit und/oder einem „echten Problem“ aus ihrem Leben über die Grenzen des Sprachunterrichts hinaus, indem ihre subjektiven Interessen, ihr Vorwissen und ihre Erfahrungen nicht nur in die Projektplanung, sondern auch in den gesamten Prozess bis hin zum Projektergebnis mitbestimmend eingebunden wurden. 
KIND, Anette; Scholz, Ingrid; Auf Der Maur Tomé, Simone, - Videoprojekt:.. Para lá da tarefa: implicar os estudantes na aprendizagem de línguas estrangeiras no ensino superior. Porto: FLUP, 2019, pp. 226-244 DOI: https://doi.org/10.21747/9789898969217/paraa12

b) ein hohes Maß an inhaltlicher und methodischer Offenheit, durch die ein Raum geschaffen wurde, in dem die Lernenden von Anfang an selbstverantwortlich und binnendifferenziert arbeiten konnten.

Wie oben erwähnt, lässt das straffe, am Europäischen Referenzrahmen orientierte Lernpensum kaum Raum für kreative und ein größeres Ergebnis anvisierende Tätigkeiten, die fremdsprachliches Handeln und selbstverantwortliches Arbeiten fördern. Eine Möglichkeit, diese Lücke zu schließen, sehen Funk et al. (2004) in verschiedenen Formen des projektorientierten Lehrens und Lernens, die sich als ideale Möglichkeiten anbieten „den Anspruch komplexen Lernens einzulösen, indem sie sowohl das individuelle als auch das kooperative Lernen in authentischen Zusammenhängen fördern und den Schülern [Studierenden] Gelegenheit dazu geben, Verantwortung für den eigenen Lernprozess zu übernehmen“ (Funk et al., 2014, p. 126).

Die Projektmethode von Frey (2007) stellt im deutschsprachigen Raum den bisher bekanntesten Versuch dar, Projektarbeit nach Phasen aufzugliedern (vgl. Krumm et al., 2010, p. 1173). Das Freysche Modell zielt auf handlungsorientiertes Lernen ab und hebt die Relevanz individueller Erlebnisse sowie gemeinsamer kommunikativer Reflexion der verschiedenen Prozesse hervor. Für ihn liegen die Vorteile der Projektarbeit u.a. darin, dass die Lernenden das Gelernte leichter transferieren und länger behalten, weil sie sich selbst mit der Themenfindung auseinandersetzen müssen und sich das zu Erlernende entdeckend aneignen. Frey sieht fünf klar definierte Projektphasen vor: Ausgangspunkt ist die Projektinitiative bzw. eine Phase, in der Themenvorschläge gesammelt werden. In der zweiten Phase erfolgt die Projektskizze bzw. die Auseinandersetzung mit der Projektinitiative. In dieser Phase werden Schwerpunkte gesetzt und ein organisatorischer Rahmen festgelegt. Der Projektplan wird im dritten Schritt entwickelt, in dem das gemeinsame Betätigungsfeld und der Projektverlauf geplant werden. Im Anschluss an die Projektplanung erfolgt die Projektdurchführung. Darin soll im Idealfall ein wechselseitig wirkendes Zusammenspiel von Projektplan und -durchführung bestehen. Die letzte Phase besteht in einem bewussten Projektabschluss durch Rückkoppelung zur Projektinitiative, Reflexion und Evaluation.

In Anlehnung an die Projektmethode von Frey schlägt Stoller (2002, p. 112) ein ähnliches Modell mit Fokus auf den fremdsprachlichen Unterricht vor, das auf zehn Schritten aufbaut: 1. Thema verabreden, 2. Zielsetzung verabreden, 3. Projekt strukturieren, 4. Schritt 5 sprachlich vorbereiten, 5. Informationen einholen, 6. Schritt 7 sprachlich vorbereiten, 7. Informationen analysieren und aufbereiten, 8. Schritt 9 sprachlich vorbereiten, 9. Ergebnisse präsentieren, 10. Evaluieren. Stoller selbst weist 
KIND, Anette; Scholz, Ingrid; Auf Der Maur Tomé, Simone, - Videoprojekt:..

Para lá da tarefa: implicar os estudantes na aprendizagem de línguas estrangeiras no ensino superior. Porto: FLUP, 2019, pp. 226-244 DOI: https://doi.org/10.21747/9789898969217/paraa12

darauf hin, dass diese zehn Schritte nicht als starres Rezept zu verstehen sind, damit die Projektarbeit nicht auf eine Technik reduziert wird, „was leicht zu routinehaftem und monotonem Unterricht führen kann“ (Krumm et al., 2010, p. 1174).

Auch Lehker (2003, p. 564) entwickelt als Erweiterung der Freyschen Grundlage ein integratives Modell von Projektarbeit, das - im Gegensatz zu linearen Projekten - ein wechselwirkendes Zusammenspiel verschiedener Projektphasen (Strukturierung, Verzahnung, Themenwahl sowie Erkundungen / Erforschungen) zugrundlegt. In ihrem integrativen Ansatz steht am Anfang des Projekts die Problemfindung: Lehrende und Lernende einigen sich auf die Bearbeitung eines Themas. In diesem Schritt planen sie zusammen das weitere Vorgehen und stellen den Bedarf an Wissen und Können fest, um das Problem bewältigen zu können. In den weiteren Phasen erwerben die Lernenden in Lehrgangs- und Übungsphasen (im Unterricht) sowie durch Erkundungen oder Erforschungen (außerhalb des Klassenraums) das notwendige Wissen, um das Projekt zu bewältigen. Der Lernprozess findet demnach sowohl im Rahmen des Fremdsprachenunterrichts als auch außerhalb des Unterrichtsraums statt (z.B. durch Interviews oder Recherchearbeit). Lernprozess und Projektdurchführung erfolgen parallel, indem sprachliche Strukturen, die für die Umsetzung des Projekts notwendig sind, im Laufe des Prozesses geübt werden. Dieses für die Durchführung von Projekten im deutschsprachigen Raum von Lehker entwickelte Modell zeichnet sich ferner durch die Interaktion der Lernenden mit dem sozialen Umfeld aus.

\section{3 - Modellmodulation für den Videowettbewerb „Mein geheimes Porto“}

Da unser Projekt nicht im Zielsprachenland durchgeführt wurde, ließ sich keins der drei beschriebenen Modelle ohne weiteres als Basis für die Projektstruktur anwenden, denn die Informationen, die in den sogenannten Erkundungs- und Erforschungsphasen erhoben wurden, u.a. Informationsgänge in der Muttersprache Portugiesisch und Gespräche mit Menschen, die der Zielsprache nicht mächtig sind, der sprachlichen Verarbeitung bzw. Übersetzung bedurften, was nicht nur die Anwendung angemessener Redemittel, sondern auch Sprachkenntnisse zur räumlichen und zeitlichen Situierung voraussetzte. So dienten etwaige Handlungsräume außerhalb des Unterrichtsraums nicht der Interaktion in der Fremdsprache, sondern als Grundlage für a) sprachliche Handlungsarbeit, b) inhaltliche Gestaltung und c) aktive Handlungsdurchführung angesichts des sprachlich-kommunikativen Ziels, nämlich der Aufgabe, geheime Orte der Stadt auf Deutsch in Kurzvideoform vorzustellen. 
KIND, Anette; Scholz, Ingrid; Auf Der Maur Tomé, Simone, - Videoprojekt:..

Para lá da tarefa: implicar os estudantes na aprendizagem de línguas estrangeiras no ensino superior.

Porto: FLUP, 2019, pp. 226-244

DOI: https://doi.org/10.21747/9789898969217/paraa12

Davon ausgehend, dass Projekte im Fremdsprachenunterricht in einzelne, klar bestimmbare Phasen gegliedert sind, die aber je nach Gegebenheiten (Lernkontext, Lernergruppe etc.) modifiziert werden können (und sollen), entwickelten wir auf der Grundlage der hier angerissenen theoretischen Ansätze folgendes Projektmodell:

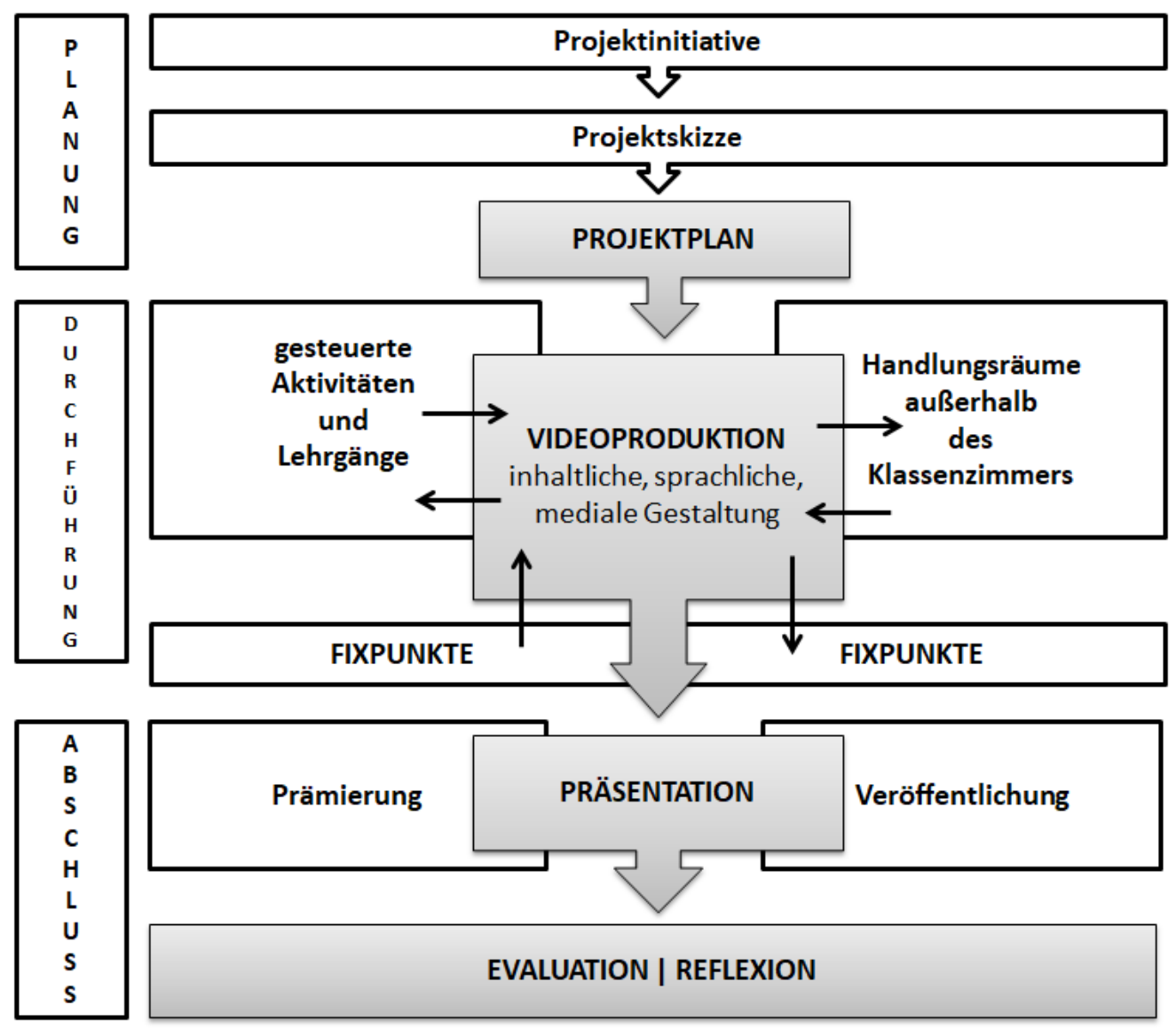

$A b b$. 1. Struktur zum Videoprojekt „Mein geheimes Porto“ | eigenes Modell in Anlehnung an die theoretischen Grundlagen von Lehker 2003, Frey 2007 und Stoller 2002.

\section{1 - Von der Projektinitiative bis zum Projektplan}

Aus Abbildung 1 geht hervor, dass sich unser Projekt in die drei Phasen Planung (Schritte im Vorfeld), Durchführung (konkrete Arbeit am Projekt und begleitende Initiativen) und Abschluss (Präsentation und Reflexion) gliederte.

In der Planungsphase wurden aufgrund der oben erwähnten zeitlichen Rahmenbedingungen Konzept und Ziele des Projekts durch die Dozentinnen bestimmt. Die Ausschreibung zur Teilnahme erfolgte im Februar 2017 per Bekanntmachung über den institutionellen E-Mail-Verteiler, über das fakultätsinterne Projektbüro und über ein zu diesem Zweck erstelltes Poster, auf dem sich neben der Vorstellung und den Eckdaten zum Projekt ein QR-Code befand, durch den genauere Informationen zu Zielpublikum, Format der Beiträge, Videoworkshop, Prämierungskriterien, 
KIND, Anette; Scholz, Ingrid; Auf Der Maur Tomé, Simone, - Videoprojekt:.. Para lá da tarefa: implicar os estudantes na aprendizagem de línguas estrangeiras no ensino superior. Porto: FLUP, 2019, pp. 226-244 DOI: https://doi.org/10.21747/9789898969217/paraa12

Abschlussveranstaltung mit Siegerehrung und Hinweis auf geplante Publikationen der prämierten Kurzvideos sowie ein detaillierter Zeitplan der diversen Projektphasen und der damit zusammenhängenden Initiativen eingesehen werden konnten. Von den insgesamt rund 300 an der Fakultät eingeschriebenen Studierenden mit Deutsch als einem der Pfeiler des Studienplans nahmen 72 Studierende aus den Niveaustufen A1 bis C1 am Wettbewerb teil. In den nächsten Schritt der Planungsphase (Projektskizze) wurden die Studierenden aktiv involviert: Auf Grundlage der Initiative und der Vorgaben der Projektausschreibung erfolgten ein Brainstorming und eine Ideensammlung möglicher Orte und potenzieller Objekte. Darauf folgten die konkrete inhaltliche Bestimmung des Projektthemas, die Konzeptentwicklung der zu erzählenden Geschichte zu diesem Ort/Objekt, die Auseinandersetzung mit den beabsichtigten Zielen und die Zeitplanung (Konzept, Recherche und Durchführung). Der Leitfrage folgend „Wie wollen wir die Projektidee umsetzen?" erarbeiteten die Teilnehmer*innen in selbstverantwortlicher Teamarbeit einen genauen Arbeits- und Zeitplan (Projektplan). Auch die Aufgaben wurden unter den Teammitgliedern gemäß ihren spezifischen Kompetenzen verteilt: Medienkompetenz, sprachliche Kompetenz und Ortskundigkeit.

\section{2 - Projektdurchführung}

Dieser konkrete Projektplan erfuhr seine praktische Umsetzung in der Phase der Projektdurchführung, die von den Dozentinnen organisierte Aktivitäten sowie eigenständige Handlungsfelder der Projektteilnehmenden außerhalb des Unterrichts beinhaltete.

$\mathrm{Zu}$ den gesteuerten Aktivitäten gehörten ein Workshop für die Bearbeitung von Videos sowie ein von einer Praktikantin angebotenes Tutorium. In dem dreistündigen, von einer Medienwissenschaftlerin und Filmemacherin durchgeführten Workshop (März 2017) wurden den Teilnehmer*innen einerseits Hinweise für den Umgang mit Kamera, Laptop, Mobiltelefon, Mikrofon und Stativ, andererseits Hinweise zu Aufnahmetechniken oder juristischen Fragen wie der Verwendung von Musikvorlagen gegeben. Schwerpunktmäßig wurde eine spezifische Software zur Bearbeitung von Videos (Windows Movie Maker) eingeführt und die Studierenden wurden im Umgang damit, insbesondere mit Schnitt- und Montagetechniken geschult.

In den Sitzungen des Tutoriums konnten sie Fragen zur sprachlichen Ausgestaltung ihrer Beiträge mit Hilfe einer deutschen Muttersprachlerin klären sowie die Aussprache der Begleittexte zu ihren Videos üben. Das Tutorium war ein Angebot für alle Teilnehmer*innen und erfolgte auf freiwilliger Basis. Parallel dazu wurden beide 
KIND, Anette; Scholz, Ingrid; Auf Der Maur Tomé, Simone, - Videoprojekt:.. Para lá da tarefa: implicar os estudantes na aprendizagem de línguas estrangeiras no ensino superior. Porto: FLUP, 2019, pp. 226-244 DOI: https://doi.org/10.21747/9789898969217/paraa12

Vorgänge durch festgelegte Austauschmomente zwischen Lernenden und Lehrenden unterstützt. Diese Fixpunkte dienten der Besprechung der Wettbewerbskriterien, der Klärung inhaltlicher, sprachlicher, technischer und organisatorischer Fragen sowie der Beantwortung von Fragen zum Script oder zum Zeitplan.

$\mathrm{Zu}$ den Handlungsräumen außerhalb des Klassenraums, in denen die Projektteilnehmenden autonom arbeiteten, gehörte neben Informationsgängen und Stadterkundungen auch die Materialbeschaffung (Recherche im Internet, Interviews, Videoaufnahmen). Zur inhaltlichen und sprachlichen Gestaltung der Videobeiträge verfassten die Studierenden eine schriftliche Vorlage, das Skript, in dem die Sequenz der Videoaufnahmen die Begleittexte, Sprecherwechsel und andere strukturierende Elemente festgelegt wurden. Diese schriftliche Vorlage diente im nächsten Schritt als Grundlage der medialen Gestaltung, die unter Berücksichtigung der im Workshop gegebenen Hinweise von den Teilnehmer*innen in selbständiger Arbeit vorgenommen wurde.

Die gesteuerten Aktivitäten und die Handlungen außerhalb des Unterrichts / Interaktion in/mit der (Um-)Welt des Lernenden fanden, wie bereits erwähnt, in stetigem Wechsel statt, so dass es zu Synergieeffekten zwischen beiden Handlungsfeldern kam.

\section{3 - Ergebnispräsentation}

In der Abschlussphase des Projekts wurden die Videobeiträge eingereicht und von der Jury nach einem zuvor erstellten Kriterienkatalog bewertet. Die Gewinnerbeiträge wurden in einer Abschlussfeier gezeigt und die Veröffentlichung aller Beiträge auf Online-Plattformen in die Wege geleitet.

Die Motivation und die positive Resonanz bei den Studierenden spiegelten sich in der hohen Beteiligung wider. Insgesamt wurden rund 40 Videos aus den Niveaustufen A1 bis $\mathrm{C} 1$ eingereicht. Einem zuvor erstellten Kriterienkatalog folgend (Anlage A), wählte dann die Jury (i.e. die drei projektbetreuenden Dozentinnen) insgesamt elf Gewinnervideos aus: ein Video aus der Niveaustufe C1, acht aus B1/B2 und drei aus A2/A1. Die Anzahl der prämierten Videos in den Unterkategorien ergab sich aus der stark differierenden Teilnehmer*innenzahl der verschiedenen Niveaustufen.

Kriterien für die Beurteilung der Videos waren: Originalität, sprachliche Gestaltung, Umsetzung der appellativen und kommunikativen Funktion, Informationsgehalt, Medienkompetenz und Gestaltung/Strukturierung der Kurzvideos (s. Anlage A). Für jedes Kriterium wurden nach bestimmten Leitfragen Punkte verteilt, wobei sich unsere Schlussbeurteilung jedoch nicht nur als Summe aller Faktoren und erreichten Punkte 
KIND, Anette; Scholz, Ingrid; Auf Der Maur Tomé, Simone, - Videoprojekt:.. Para lá da tarefa: implicar os estudantes na aprendizagem de línguas estrangeiras no ensino superior. Porto: FLUP, 2019, pp. 226-244 DOI: https://doi.org/10.21747/9789898969217/paraa12

betrachten ließ, da wir am Ende in einer ausführlichen Auswahldiskussion die Beurteilung gewisser Beiträge aufgrund des Gesamteindrucks ggf. leicht anders positionierten.

In einer Abschlussfeier im akademischen Umfeld erfolgte nach Ende der Vorlesungszeit (Juni 2017) die Siegerehrung mit Präsentation der prämierten Videos und Preisverleihung. In Anwesenheit institutioneller Vertreter, u.a. der Dekanin, der Abteilungsleitung, der Kolleg*innen und Student*innen, wurden die Beiträge gebührend gewürdigt. Die Autor*innen der Gewinnervideos erhielten Preise zu deutscher Sprache, Literatur und Kultur: Zeitschriftabonnements, Videos, Bücher, Musik, Grammatiken und Spiele, die vom Deutschen Akademischen Austauschdienst (DAAD) sowie dem Spotlight Verlag finanziert wurden. Im Anschluss wurde, wie es sich für die Stadt Porto gehört, ein Umtrunk mit Portwein gereicht.

Die Gewinnervideos wurden in einer späteren Phase auf diversen Plattformen veröffentlicht: auf der Internetseite der Fakultät, der Universität Porto sowie der Deutschen Botschaft in Lissabon unter der Rubrik Kultur und Bildung. ${ }^{2}$

\subsection{1 - Evaluation und kritische Auswertung}

Dass sich einige Schritte, die wir ursprünglich eingeplant hatten, nicht realisieren ließen, war im Wesentlichen dem engen Zeitrahmen sowie der Ausgliederung des Projekts aus dem Unterrichtsverlauf geschuldet.

So hatten wir beispielsweise daran gedacht, die Studierenden an der Auswahl der Gewinnervideos durch ein Online-Voting aktiv einzubeziehen und sie dadurch neugierig auf die Beiträge der anderen zu machen. Da wir jedoch den Abgabetermin auf Wunsch der Teilnehmer*innen verschieben mussten und die Abschlussveranstaltung aus logistischen Gründen direkt nach Vorlesungsende stattfinden sollte, ließ sich dieses nicht mehr umsetzen.

Weiterhin kam es zwischen Abschlussveranstaltung und Veröffentlichung nicht mehr zu einer selbständigen Überarbeitung einiger Videos, nachdem wir die Studierenden darauf aufmerksam gemacht hatten, dass ihre Beiträge durch eine erneute Bearbeitung von Ton oder Bild oder durch sprachliche Korrekturen an Qualität gewinnen würden, da sie sich bereits in der Examensphase befanden und dafür keine Zeit mehr fanden. So beobachteten wir auch unter den Gewinnervideos kleinere Mängel in Ton- und

\footnotetext{
2 https://lissabon.diplo.de/pt-de/themen/kultur/uni-porto-germanistik-2017/1717646 https://sigarra.up.pt/flup/pt/noticias_geral.ver_noticia?p_nr=72243 https://noticias.up.pt/estudantes-de-alemao-da-flup-revelam-o-lado-secreto-do-porto/
} 
KIND, Anette; Scholz, Ingrid; Auf Der Maur Tomé, Simone, - Videoprojekt:..

Para lá da tarefa: implicar os estudantes na aprendizagem de línguas estrangeiras no ensino superior. Porto: FLUP, 2019, pp. 226-244 DOI: https://doi.org/10.21747/9789898969217/paraa12

Bildqualität. Zum Beispiel war in „Der literarischer Porto“, der als einziger Beitrag den Spuren der Portuenser Schriftsteller nachgeht, die Stimme aus dem Off zu leise. In „Espaço STOP“ hatten die zwei Studentinnen den Einleitungstext auf der Straße bei starkem Wind gesprochen, was zu starkem Rauschen führte und die Verständlichkeit beeinträchtigte. Die Studentinnen fanden zwar eine Notlösung, indem sie Untertitel in der gleichen Sprache (Deutsch) einfügten, dieses hätte sich jedoch ebenfalls durch eine Neuaufnahme vermeiden lassen. In „Tripeiros“, einem Beitrag, der sich dem typischen Lokalgericht der Stadt und seiner kuriosen Entstehungsgeschichte widmet, führt der zu schnelle Bildwechsel dazu, dass sich der Zuschauer kaum auf ein Bild einstellen kann. Hier hätte eine andere Form der Montage Abhilfe schaffen können.

Daher sind wir zu dem Schluss gekommen, dass der Workshop zur Bearbeitung von Videos vor dem anvisierten Ziel der Förderung medialer Kompetenz in zwei Phasen hätte stattfinden sollen: Eine Phase, in der die Grundlagen für den Umgang mit Hardund Software gelegt werden, und eine zweite Phase, in der die bereits gedrehten Szenen in Montage, Bild- und Tonbearbeitung hätten betreut werden können. Der von uns angebotene Workshop zur Videobearbeitung ermöglichte hingegen nur einen Einblick in die vielfältigen technischen Möglichkeiten und eine kurze Übungsphase; insbesondere Hinweise zur Tongestaltung konnten nur angerissen werden.

Ebenso fehlte eine gemeinsame kritische Auswertung durch die das Projekt betreuenden Dozentinnen und die teilnehmenden Studierenden. Es war zwar während der Projektarbeit, bei der Abschlussveranstaltung und auch in punktuellen Gesprächen danach offensichtlich, dass die teilnehmenden Studierenden mit großem Enthusiasmus mitgearbeitet hatten, dennoch fehlte ein Austausch über Themenfindung, Projektverlauf und ein Feedback über ursprüngliche Erwartungen und tatsächliche Ergebnisse in Hinsicht auf die inhaltliche, sprachliche und mediale Gestaltung.

Dies wäre in einem Folgeprojekt mit einem ähnlichen Ansatz wünschenswert, zumal die eingereichten Beiträge den Erwartungen der Dozentinnen hinsichtlich des "Besonderen“ und "Geheimen“ im Wesentlichen überraschend oft nicht ganz entsprachen. Diese Diskrepanz zwischen unseren Erwartungen und dem, was die Studierenden de facto als besonders und „geheim“ empfanden, kritisch miteinander zu reflektieren, hätte voraussichtlich zu einem Erkenntnisgewinn hinsichtlich kultureller Unterschiede und einer Sensibilisierung für eben diese Unterschiede auf beiden Seiten geführt. Für uns stellte sich als unerwartetes Ergebnis heraus, dass die portugiesischen Studierenden, die wir an unserer Hochschule unterrichten, präsentationswürdige "geheime“ Orte oder Details ihrer Studienstadt offenkundig nach anderen Kriterien 
KIND, Anette; Scholz, Ingrid; Auf Der Maur Tomé, Simone, - Videoprojekt:..

Para lá da tarefa: implicar os estudantes na aprendizagem de línguas estrangeiras no ensino superior. Porto: FLUP, 2019, pp. 226-244 DOI: https://doi.org/10.21747/9789898969217/paraa12

aussuchten, als wir es erwartet hätten. Nicht nur das Konzept des „Originellen“, sondern auch das des „Geheimen“ wurden anders als erwartet umgesetzt: Einige Beiträge konzentrierten sich nicht, wie vorgegeben, auf ein Objekt bzw. einen Ort, sondern führten den Zuschauer quer durch die Stadt von einer Sehenswürdigkeit zur anderen, so dass sich diese Beiträge als Sammelsurium von "geheimen“ Orten erwiesen, in denen der Fokus auf ein Objekt fehlte. Diese haben wir bei der Auswahl der zu prämierenden Videos von vornherein ausgeschlossen. Andere wiederum stellten Orte vor, die jedem Portuenser vertraut sind und somit nicht mehr unter die Kategorie "geheim" fallen dürften. Bei Nachfragen stellte sich heraus, dass die betreffenden Studierenden gar nicht aus Porto stammten und sich ihre Studienstadt bis dato auch noch nicht ausreichend erschlossen hatten, um in der Lage zu sein, Geheimtipps weiterzureichen. Dies betrifft im Übrigen einen Großteil unserer Studierenden, und selbst denjenigen, die aus der unmittelbaren Umgebung Portos kommen, ist diese Stadt nur unzureichend vertraut. Dies führte dazu, dass die Projektteilnehmenden teils Orte vorstellten, die zwar in jedem Reiseführer angepriesen werden, die sie aber für sich persönlich bei einem Stadtrundgang „entdeckt“ hatten. Als Geheimtipp angepriesen wurde beispielsweise der traditionelle Feinkostladen „A Pérola do Bolhão“ im Herzen der Stadt, der de facto mit einer beeindruckenden Jugendstil-Kachelfassade und einem überwältigenden Angebot an Delikatessen und regionalen Spezialitäten aufwarten kann, eigentlich aber in das Standardprogramm jedes Portobesuchers gehört. Da unser erstes Bewertungskriterium, das daher auch die stärkste Gewichtung bei der Punkteverteilung erhielt, der Aspekt "Originalität“ war, lag es nahe, diese Beiträge abzustufen oder gegebenenfalls ganz von der Beurteilung auszuschließen. Bei der Auswertung wurde uns allerdings bewusst, dass unsere Studierenden offenkundig von einem ganz anderen Konzept von „Originalität“ oder „Geheimtipp“ ausgegangen waren, so dass solche Beiträge uns als das Projekt betreuende Dozentinnen und Jury in Personalunion vor die schwierige Frage der Beurteilung stellten.

In diesem Zusammenhang wurde auch deutlich, dass die geographische wie auch soziale Herkunft der Studierenden bei der Objektauswahl eine nicht zu unterschätzende Rolle spielten: Während die Studierenden unserer Fakultät vor etlichen Jahren noch vorrangig aus dem Portuenser Bildungsbürgertum stammten, haben wir es jetzt nicht nur vermehrt mit Studierenden aus der Peripherie oder nordportugiesischen Kleinstädten zu tun, sondern auch das soziale Spektrum der Herkunftsfamilien scheint uns weiter gefächert zu sein, worüber allerdings keine genauen Erhebungen vorliegen. 
KIND, Anette; Scholz, Ingrid; Auf Der Maur Tomé, Simone, - Videoprojekt:.. Para lá da tarefa: implicar os estudantes na aprendizagem de línguas estrangeiras no ensino superior. Porto: FLUP, 2019, pp. 226-244 DOI: https://doi.org/10.21747/9789898969217/paraa12

Des Weiteren stellte sich heraus, dass, von wenigen Ausnahmen abgesehen, die vorgestellten Objekte mit den Attributen schön, groß, geschichtsträchtig oder imposant zu bezeichnen sind: große Parkanlagen, alte herrschaftliche Bauten oder gemütliche Gartencafés. Von wenigen Ausnahmen abgesehen hatten sich die Teilnehmer*innen ein Objekt im Zentrum der Stadt ausgesucht - Orte in der Peripherie schienen innen von vornherein nicht mit der notwendigen Repräsentativität ausgestattet zu sein, um in einem Beitrag, der ja später auch von einem deutschen Publikum rezipiert werden sollte, vorgestellt zu werden.

Aus der Auswertung der Beiträge ergab sich, dass Skurriles, Ausgefallenes, Exzentrisches, Schrilles, Groteskes, Bizarres, Kurioses, was wir Dozentinnen (zwei Deutsche und eine Deutsch-Schweizerin) durchaus wertschätzen, von den meisten unserer Studierenden nicht als vorzeigbar bzw. präsentationswürdig erachtet wurde. Somit ließen sich eklatante Unterschiede in der Wahrnehmung seitens der Dozentinnen und der Studierenden konstatieren: Für unseren Blick (den deutsch-schweizerischen Blick einer älteren Generation, die eine andere Sozialisation an einem anderen Ort unter anderen kulturellen Vorzeichen erfahren hat) sind o.g. Attribute durchaus positiv konnotiert, nicht aber für die Studierenden, die Objekte mit diesen Eigenschaften allem Anschein nach eher als abwegig, befremdlich oder unkonventionell wahrnehmen und daher eindeutig negativ besetzen. Das Alte, Hässliche, Verfallene oder Dekadente kam in den Kurzvideos beispielsweise so gut wie gar nicht vor, was die Schlussfolgerung nahelegt, dass unsere Studierenden für den Charme des Vergänglichen und Morbiden recht unempfänglich sind. Es ist davon auszugehen, dass diese Diskrepanzen in Wahrnehmungsweise und Wertschätzung von urbanen Elementen vorrangig kulturell bedingt ist, sekundär aber wohl auch generationenbedingt sein mag. Aufschluss darüber könnte nur ein Kontrollprojekt geben, worauf wir im Ausblick näher eingehen werden.

\section{4 - Ausblick}

Wie ließe sich auf den aus der kritischen Reflexion gewonnenen Erkenntnissen aufbauend weiterarbeiten? Was könnte man bei einem zukünftigen Projekt in dieser Form übernehmen, was anders machen?

Wie bereits dargestellt, erwiesen sich sowohl das Projektthema wie das Medium Kurzvideo als stark motivationsfördernd. Einerseits konnten die Projektteilnehmenden aus ihrer persönlichen Sicht etwas aus ihrer Studienstadt vorstellen, waren also 
KIND, Anette; Scholz, Ingrid; Auf Der Maur Tomé, Simone, - Videoprojekt:..

Para lá da tarefa: implicar os estudantes na aprendizagem de línguas estrangeiras no ensino superior. Porto: FLUP, 2019, pp. 226-244 DOI: https://doi.org/10.21747/9789898969217/paraa12

individuell und emotional involviert, andererseits erwies sich das Medium Film als geeignet, da es ein ganzheitliches Arbeiten erlaubte (Zusammenspiel von Bild, Ton, Musik, geschriebener und gesprochener Sprache ebenso wie eine wechselseitige Dynamik verschiedener sprachlicher Fertigkeiten) und den Neigungen einer medienerfahrenen Generation nicht nur entgegenkam, sondern ihre Medienkompetenz auch erweiterte. Auch der konkrete Kommunikationsanlass, d.h. die Aussicht, ihre Kurzfilme später auf diversen Plattformen veröffentlicht zu sehen und damit auch deutschsprachige Portobesucher*innen direkt ansprechen zu können, diente der Motivationssteigerung.

Im vorangehenden Kapitel, das der kritischen Auswertung gewidmet ist, wurden bereits ausführlich etliche Aspekte beleuchtet, die aufgrund unserer Erfahrung anders gestaltet werden könnten, wohlgemerkt unter der Voraussetzung, dass man über ausreichend Zeit und die notwendigen finanziellen Mittel verfügt.

Um oben angeführte Diskrepanzen zwischen Erwartungen der Projektleiterinnen und den tatsächlichen Ergebnissen abzufedern, würden wir empfehlen, in der Vorbereitungsphase trotz allen Zeitmangels nicht auf eine gemeinsame Bestimmung, d.h. seitens der Lehrenden und der Lernenden, von Konzept, Erwartungen und Zielen des Projekts zu verzichten. In der Durchführungsphase könnte eine intensivere Betreuung des Entstehungsprozesses geboten werden, was allerdings einen höheren Zeitaufwand bzw. die Einbindung des Projekts ins Unterrichtsgeschehen zu Lasten anderer curricularer Programmpunkte bedeuten würde. In diesem Zusammenhang wäre die Thematisierung der Begriffe von "geheim" „und "originell“ von grundlegender Bedeutung. Auch die sprachliche Gestaltung könnte - vorausgesetzt, man hätte die Zeit dazu - durch die Korrektur der Texte und das Üben von Aussprache, Diktion und Prosodie im Unterricht intensiver betreut werden.

In der Nachbereitungsphase sollte eine gemeinsame Nachbesprechung und kritische Reflexion der Ergebnisse stattfinden, um Antworten auf die Frage zu finden, wie sich die unterschiedliche Wahrnehmungsweise vonseiten der Lektorinnen und der Studierenden erklären lässt und um eine Sensibilisierung der Studierenden (und der Dozentinnen) für kulturelle Unterschiede zu erreichen.

Auf Grundlage der kritischen Auswertung könnte man in einem Folgeprojekt versuchen, festgestellte Schwächen durch erwähnte Projektschritte zu beheben. Als aufschlussreich könnte sich auch eine Kontrollstudie in Zusammenarbeit mit Dozenten und Studierenden (beispielsweise der Lusitanistik) einer deutschen Hochschule erweisen. Ein gemeinsames Projekt zu einem ähnlichen Thema (portugiesische 
KIND, Anette; Scholz, Ingrid; Auf Der Maur Tomé, Simone, - Videoprojekt:.. Para lá da tarefa: implicar os estudantes na aprendizagem de línguas estrangeiras no ensino superior. Porto: FLUP, 2019, pp. 226-244 DOI: https://doi.org/10.21747/9789898969217/paraa12

Studierende stellen geheime Orte/Objekte ihrer Stadt auf Deutsch vor, deutsche Studierende stellen geheime Orte/Objekte ihrer Stadt auf Portugiesisch vor) könnte Aufschluss darüber geben, inwiefern die in der kritischen Auswertung genannten Diskrepanzen hinsichtlich der Konzepte von „Geheimem“ und „Originellem" tatsächlich kulturell bedingt sind und inwiefern, falls sich ähnliche Tendenzen feststellen ließen, diese auch auf eine generationsbedingte Sensibilität europäischer Student*innen zurückzuführen wären.

Weiterhin wäre es interessant, Erfahrungen mit Dozent*innen auszutauschen, die mit ihren Studierenden ein ähnliches Projekt, vielleicht in Anlehnung an das hier dargestellte Modell und die hier zum Einsatz gekommene Thematik aufgreifend, durchführen würden, um herauszufinden, wie es in anderen Ländern um die Auffassung dessen, was als originell, geheim und daraus folgend als präsentationswürdig empfunden wird, bestellt ist.

Abschließend möchten wir festhalten, dass sich der gesamte Projektverlauf für alle Beteiligten als sehr bereichernd erwies. Für uns gehörte dazu die Erkenntnis, dass auch in einem im Vorfeld geplanten Projekt Unvorhergesehenes eintreten kann, insbesondere wenn Akteure mit unterschiedlicher kultureller Prägung involviert sind.

\section{Literatur}

Apeltauer, E. (1997). Grundlagen des Erst- und Fremdsprachenerwerbs. Eine Einführung. Fernstudieneinheit 15, Kassel: Langenscheidt.

Baacke, D. (1996): Medienkompetenz als Netzwerk. Reichweite und Fokussierung eines Begriffs, der Konjunktur hat. In: medien praktisch 2, 4-10.

Baacke, D. (1999): Medienkompetenz als zentrales Operationsfeld von Projekten. In: Baacke, Dieter u.a. (Hrsg.): Handbuch Medien: Medienkompetenz. Bonn: Bundeszentrale für politische Bildung, 31-35.

Barkowski, H. \& Krumm, H.-J. (Hrsg.) (2010). Fachlexikon Deutsch als Fremd- und Zweitsprache. (UTB 8422). Tübingen: A. Francke.

Ehlich, K. (2007): Sprache und sprachliches Handeln. Pragmatik und Sprachtheorie, Prozeduren des sprachlichen Handelns, Diskurs, Narration, Text, Schrift. Berlin, New York: De Gruyter.

Frey, K. (2007): Die Projektmethode. Der Weg zum bildenden Tun. Weinheim/Basel: Beltz.

Funk, H., Kuhn, C., Skiba, D., Spaniel-Weise, R. \& Wicke, E. (2014) (Hrsg). DLL 04: Aufgaben, Übungen, Interaktion. Stuttgart: Ernst Klett Sprachen. 
KIND, Anette; Scholz, Ingrid; Auf Der Maur Tomé, Simone, - Videoprojekt:.. Para lá da tarefa: implicar os estudantes na aprendizagem de línguas estrangeiras no ensino superior. Porto: FLUP, 2019, pp. 226-244 DOI: https://doi.org/10.21747/9789898969217/paraa12

Gudjons, H. (2001). Handlungsorientiert lehren und lernen. 6., überarb. u. erw. Auflage, Bad Heilbrunn.

Mandl, H. \& Gräsel, C. (1997). Multimediales und problemorientiertes Lernen. Thyroidea - ein Lernprogramm für das Medizinstudium. In: Hamm, I. \& Müller-Böling, D. (Hrsg.). Hochschulentwicklung durch neue Medien. Gütersloh, S. 173-183.

Jank, W. \& Meyer, H. (2000): Didaktische Modelle. 7. Auflage, Berlin.

Kleppin, K. (2004). Bei dem Lehrer kann man ja nichts lernen. Zur Unterstützung von Motivation durch Sprachlernberatung. Zeitschrift für Interkulturellen Fremdsprachenunterricht [Online], 9(2). URL: http://www.ualberta.ca/ german/ejournal/Kleppin2.htm (11.03.2019).

Krumm H.-J., Fandrych, C., Hufeisen, B. \& Riemer, C. (Hgg.) (2010). Handbücher zur Sprach- und Kommunikationswissenschaft / HSK. Band 2 (2. und vollständig überarbeitete und neu zusammengestellte Auflage). Berlin/New York: de Gruyter.

Legutke, M. (2003): „Projektunterricht“. In: Bausch, Karl-Richard et. al: Handbuch Fremdsprachenunterricht. 4. Auflage. Tübingen: Francke.

Peuschel, K. (2012). Sprachliche Tätigkeit und Fremdsprachenlernprojekte. Fremdsprachliches Handeln und gesellschaftliche Teilhabe. in radiodaf-Projekten. Baltmannsweiler: Schneider-Verlag Hohengehren.

Schwerdtfeger, I. (1997). Der Unterricht Deutsch als Fremdsprache: Auf der Suche nach den verlorenen Emotionen, Info DaF, 24(5), 587-606.

Stoller, F. (2002): Project work: a means to promote language in content. In: Richards, J. \& Renandya, W. (Hg.), Methodology in Language Teaching, 107-120. Cambridge: Cambridge University Press. 


\section{Anhang A}

\section{Kriterien für die Beurteilung der Videos „Mein geheimes Porto“ FLUP 2016/17 (Anette Kind - Ingrid Scholz - Simone Tomé)}

\section{Originalität}

a) das Objekt per se: fernab des

Mainstreams ?

b)

Überraschun $\mathrm{g}+$

Authentizität:

in

Bezug auf die Art und Weise, wie das Objekt präsentiert wird

- waru

$\mathrm{m}$ dieses

Thema?

Geht das persönliche Interesse/ die Begeisterung aus dem Film hervor?

$\begin{array}{ll}\text { sprachliche } & \text { Kommunik } \\ \text { Gestaltung } & \text { a-tive } \\ & \text { Funktion } \\ & \text { erreicht? }\end{array}$

$$
1 \text { - } 6
$$

1 - 4

1 - 4

1 - 4

\begin{tabular}{|c|c|}
\hline a) Korre & (unabhängi \\
\hline ktheit & g von der \\
\hline b) lexika & sprachliche \\
\hline lische & $\mathrm{n}$ \\
\hline $\begin{array}{l}\text { Angemes- } \\
\text { senheit }\end{array}$ & Korrektheit) \\
\hline c) Auss & \\
\hline prache & Kommt die \\
\hline $\begin{array}{l}\text { (korrekt, } \\
\text { laut und }\end{array}$ & $\begin{array}{l}\text { Botschaft } \\
\text { an? }\end{array}$ \\
\hline
\end{tabular}
deutlich)

d) Inton ation/ Prosodie

Hintergrund-
wissen: Wie
viel
Recherche
wurde
durchgeführt
?

?

\section{Handhabung} von/Umgang mit Kamera,

Mikrofon,

Software

(schwierig, den

Eigenbeitrag einzuschätzen )

Punktabzug bei:

a)

rauschendem
Ton

b) verwackelten Bildern
$1-4$

1 - 2

\section{Gestaltung/ \\ Strukturierung}

Appella-

tive

Funktio

n

a)

Wird der

Zuschaue

$r$ animiert, das

Objekt zu besuchen / selbst sehen zu wollen?

Wechsel der Typologie (nicht linear)

b)

b) im Stil eines

Werden Neugier und Begeisterung beim Zuschaue sen im Wechsel c) musikalische Untermalung 